refers to cases of hereditary spastic paraplegia in children with degeneration confined to the pyramidal tracts. (Diseases of the Nervous System 4th ed, Springfield, CC Thomas, 1960, p 379).

\title{
FUCOSIDOSIS WITH DYSTONIA
}

A Canadian male child with fucosidosis and dystonia is reported from the Department of Biochemical Genetics, University of Western Ontario, London, Ontario, Canada. All milestones were delayed, he crawled at 14 months, walked by 18 months, and his developmental quotient at 27 months was 50 . At 46 months the quotient had dropped to 35. At 5 years, dystonic posturing of the left leg began, and 6 months later, he had episodes of choking, staring spells, and nocturnal apnea. At 7 years, he could not walk or talk, and the dystonia was bilateral. An angiokeratomatous rash became generalized. Cultured lymphoblasts showed absent a-fucosidase activity and protein. He was homozygous for the Q422X mutation. (Gordon BA et al. Fucosidosis with dystonia. Neuropediatrics 1995;26:325-327). (Respond: Dr BA Gordon, Department of Biochemical Genetics, CPRI, 600 Sanatorium Road, London, ON, Canada N6H 3W7).

COMMENT. Fucosidosis is a progressive neurodegenerative disease presenting in early childhood and manifested by loss of mental and motor function, with early hypotonia followed by increasing spasticity and seizures. Dystonia evident in this report had not previously been reported. Dysmorphic features and skeletal changes similar to those in mucopolysaccharidoses develop and include dwarfism, dysostosis multiplex, and visceromegaly. A skin rash occurs in those surviving childhood. The disease is caused by an autosomal recessive genetic deficiency of the lysosomal enzyme a-Lfucosidase. Seventy seven patient reports were reviewed in 1991. Menkes JH refers to two forms, type 1 without, and type 2 with angiokeratoma of the skin, particularly of the gingiva and genitalia. (Textbook of Child Neurology 3rd ed, Philadelphia, Lea \& Febiger, 19850.

\section{PURINE METABOLISM AND RETT SYNDROME}

Levels of purine and pyridine nucleotides and their metabolites were determined in erythrocytes and plasma of 31 Rett pattients and 17 age-matched controls at the Departments of Molecular Biology and Child Psychiatry, University of Siena, Children's Hospital, Siena, Italy. No difference was found in erythrocyte nucleotide concentrations, but plasma nicotinamide levels were significantly lower in Rett syndrome patients compared to controls. Erythrocyte activities of hypoxanthine phosphoribosyl transferase, adenine pbt and pbpp synthetase were also lower. The production rate of pyridine nucleotides from nicotinic acid by erythrocytes was increased in Rett patients. The significance of these metabolic changes was not determined. (Rocchigiani $\mathrm{M}$ et al. Purine and pyridine nucleotide metabolism in the erythrocytes of patients with Rett syndrome. Neuropediatrics 1995;26:288-292).

COMMENT. Despite frequent reports of various and diverse metabolic changes in patients with Rett syndrome, no consistent and diagnostic biochemical test has been identified for this disorder. The diagnosis is based on agreed clinical criteria. Alterations in nucleotide metabolism are an interesting addition to the search for a specific cause. 